\title{
THE SOCLE MODULE OF A MONOMIAL IDEAL
}

\author{
LIZHONG CHU, JÜRGEN HERZOG, DANCHENG LU*
}

\begin{abstract}
For any ideal $I$ in a Noetherian local ring or any graded ideal $I$ in a standard graded $K$-algebra over a field $K$, we introduce the socle module $\operatorname{Soc}(I)$, whose graded components give us the socle of the powers of $I$. It is observed that $\operatorname{Soc}(I)$ is a finitely generated module over the fiber cone of $I$. In the case that $S$ is the polynomial ring and all powers of $I \subseteq S$ have linear resolution, we define the module $\operatorname{Soc}^{*}(I)$ which is a module over the Rees ring of $I$. For the edge ideal of a graph and for classes of polymatroidal ideals we study the module structure of their socle modules.
\end{abstract}

\section{INTRODUCTION}

Let $(S, \mathfrak{m})$ be a Noetherian local ring $S$ with maximal ideal $\mathfrak{m}$ or a standard graded $K$-algebra $S$ over a field $K$ with maximal graded ideal $\mathfrak{m}$. Furthermore, let $I \subseteq \mathfrak{m}$ be an ideal which we assume to be graded if $S$ is standard graded. In this paper we study for each integer $m \geq 0$ the socle $\left(I^{m}: \mathfrak{m}\right) / I^{m}$ of the powers $I^{m}$ of $I$. Results in this direction can be found in [5], [8] and in [10].

It is clear that the multiplication with any $f \in I^{r}$ induces a map $\left(I^{m}: \mathfrak{m}\right) / I^{m} \rightarrow$ $\left(I^{m+r}: \mathfrak{m}\right) / I^{m+r}$, so that $\operatorname{Soc}(I)=\bigoplus_{m>0}\left(I^{m}: \mathfrak{m}\right) / I^{m}$ has the structure of a graded $\mathcal{F}(I)$-module, where $\mathcal{F}(I)=\bigoplus_{m \geq 0} I^{m} / \mathfrak{m} I^{m}$ is the fiber cone of $I$. In Proposition 1.1 we notice that $\operatorname{Soc}(I)$ is a finitely generated $\mathcal{F}(I)$-module. The proof is based on the fact that, up to a truncation, $\operatorname{Soc}(I)$ can be identified with an ideal of the associated graded ring $\operatorname{gr}_{I}(S)$ of $I$.

In this paper we are mostly interested in the module structure of $\operatorname{Soc}(I)$, when $I$ is a monomial ideal and in particular in the case when $I$ is the edge ideal of a graph or a polymatroidal ideal. In both of these cases, $I$ satisfies the Ratliff condition, which means that $\left(I^{m+1}: I\right)=I^{m}$ for all $m \geq 0$, see [12, Lemma 2.12] and 8 , Theorem 4.1]. In this case, $\operatorname{Soc}(I)$, without any truncation, may be viewed as an ideal of $\operatorname{gr}_{I}(S)$. As a consequence of Theorem 1.2, this fact can be used to show that if $G$ is a finite simple graph with connected components $G_{1}, \ldots, G_{r}$, then $\operatorname{Soc}(I(G))=\operatorname{Soc}\left(I\left(G_{1}\right)\right) \operatorname{Soc}\left(I\left(G_{2}\right)\right) \cdots \operatorname{Soc}\left(I\left(G_{r}\right)\right)$. Here, $I(H)$ denotes the edge ideal of a graph $H$.

If $I$ has a $d$-linear resolution, then, as observed in Proposition 1.4, $(I: \mathfrak{m})=$ $I+\operatorname{soc}(I)$, where $\operatorname{soc}(I)$ is an ideal generated in degree $d-1$. Hence if all powers of $I$ have linear resolution, which is the case for polymatroidal ideals, we may define

2010 Mathematics Subject Classification. Primary 13F20; Secondary 13H10.

Key words and phrases. socle module, edge ideals, polymatroidal ideals, fiber cone.

* Corresponding author. 
$\operatorname{Soc}^{*}(I)=\bigoplus_{m>1} \operatorname{Soc}\left(I^{m}\right)$, which is a graded module over the Rees ring of $I$. Note that $\operatorname{Soc}(I) \cong \operatorname{Soc}^{*}(I) / \mathfrak{m} \operatorname{Soc}^{*}(I)$.

The main result of Section 2 is Theorem [2.6, where it is shown that if $G$ is a unicyclic graph with the unique odd cycle $C_{2 k+1}$ and with edge ideal $I=I(G)$, then $\operatorname{Soc}(I) \cong \mathcal{F}(I)(-c-k)$. Here, $c$ is the number of edges which do not belong to the cycle $C_{2 k+1}$ and which are not leaves. The proof of this theorem uses in a crucial way Lemma 2.1 which follows from Theorem 6.1 and Theorem 6.7 of Banerjee in [2]. The generator of $\operatorname{Soc}(I)$ is of the form $u+I^{c+k+1}$, where $u$ is a monomial of degree $2(c+k)+1$. It is clear that for any graph the generators of $\operatorname{Soc}(I)$ are of the form $u+I^{m}$ where $u$ is a monomial of degree $\geq 2 m-1$. If the graph $G$ has more than one odd cycle, then $\operatorname{Soc}(I)$ may have generators $u+I^{m}$ with $\operatorname{deg}(u)>2 m-1$, see Example 2.3. On the other hand, it is shown in Proposition 2.2 that if any two odd cycles of $G$ have distance at most one, then each monomial $u \in\left(I^{m}: \mathfrak{m}\right) \backslash I^{m}$ has degree $2 m-1$. Section 2 closes with an application of our results to the depth stability index of the edge ideal of a connected nonbipartite graph, see Corollary 2.8.

Section 3 is devoted to the study of $\operatorname{Soc}^{*}(I)$ when $I \subseteq K\left[x_{1}, \ldots, x_{n}\right]$ is a polymatroidal ideal. It follows from [9, Corollary 3.5] that $\operatorname{Soc}^{*}(I) \neq 0$ if and only if the analytic spread $\ell(I)$ of $I$ is $n$, and from [8, Theorem 4.1] that the least degree of a generator of $\operatorname{Soc}^{*}(I)$ is $<n$. We have some computational evidence that a much stronger statement holds, namely that $\operatorname{Soc}^{*}(I)$ is generated in degree $<n$. The main result of Section 3 is Theorem 3.7. There it is shown that indeed $\operatorname{Soc}^{*}(I)$ is generated in degree $<n$ for a PLP-polymatroidal ideal $I$ on the ground set $[n]$. The pruned lattice path polymatroids (PLP-polymatroidals for short) were firstly introduced by J. Schweig in [15]. Then the third author of this paper in [11] observed that this class of polymatroidal ideals is given by a system of linear inequalities and they constitute a wide generalization of polymatroidal ideals with strong exchange properties whose polymatroidal ideals are essentially of Veronese type. In Proposition 3.8 those Veronese type ideals are characterized for which $\operatorname{Soc}^{*}(I)$ is equi-generated. For the proof of these results one has to analyze carefully the system of linear inequalities defining this type of polymatroids.

\section{Definition AND BASis PROperties of THE SOCle MOdUle}

We define the socle module of $I$ to be

$$
\operatorname{Soc}(I)=\bigoplus_{m \geq 0}\left(I^{m}: \mathfrak{m}\right) / I^{m}
$$

Note that $\operatorname{Soc}(I)$ is a graded $\mathcal{F}(I)$-module, where $\mathcal{F}(I)=\bigoplus_{m \geq 0} I^{m} / \mathfrak{m} I^{m}$ is the fiber cone of $I$.

Proposition 1.1. The module $\operatorname{Soc}(I)$ is a finitely generated graded $\mathcal{F}(I)$-module.

Proof. We denote by $\operatorname{gr}_{I}(S)=\bigoplus_{m>0} I^{m} / I^{m+1}$ the associated graded ring of $I$. Then the module $C(I)=\left(0: \operatorname{gr}_{I}(S) \mathfrak{m}\right)=\left\{f \in \operatorname{gr}_{I}(S): \mathfrak{m} f=0\right\}$ is a finitely generated $\operatorname{gr}_{I}(S)$-module because it is an ideal in the Noetherian $\operatorname{ring} \operatorname{gr}_{I}(S)$. Since $\left(0: \operatorname{gr}_{I}(S) \mathfrak{m}\right)$ is annihilated by $\mathfrak{m}$ and since $\mathcal{F}(I)=\operatorname{gr}_{I}(S) / \mathfrak{m g r}_{I}(S)$ it is actually a finitely generated $\mathcal{F}(I)$-module. 
The $m$ th graded component of $C(I)$ is equal to $\left(\left(I^{m}: \mathfrak{m}\right) \cap I^{m-1}\right) / I^{m}$. Ratliff [14] showed that there exists $m_{0}$ such that $\left(I^{m}: I\right)=I^{m-1}$ for all $m \geq m_{0}$. Since $\left(I^{m}: \mathfrak{m}\right) \subseteq\left(I^{m}: I\right)$, we see that $\operatorname{Soc}(I)_{\geq m_{0}}=C(I)_{\geq m_{0}}$, where for any graded module $M, M_{\geq r}$ denotes the submodule $\bigoplus_{m \geq r} M_{m}$ of $M$. This shows that $\operatorname{Soc}(I)_{\geq m_{0}}$ is finitely generated, and consequently $\operatorname{Soc}(I)$ is finitely generated as well.

We say that $I$ satisfies the Ratliff condition if $\left(I^{m+1}: I\right)=I^{m}$ for all $m \geq 0$. Let $C(I)=\left(0:_{\operatorname{gr}_{I}(S)} \mathfrak{m}\right)$ be the module introduced in the proof of Proposition 1.1. Note that $C(I)=\operatorname{Soc}(I)$, if $I$ satisfies the Ratliff condition.

Let $I_{1} \subseteq S_{1}=K\left[x_{1}, \ldots, x_{n}\right]$ and $I_{2} \subseteq S_{2}=K\left[y_{1}, \ldots, y_{m}\right]$ be monomial ideals, and let $I=\left(I_{1}, I_{2}\right) S \subseteq S$, where $S=S_{1} \otimes_{K} S_{2}$. By [4, Proposition 3.2], the canonical map $\alpha: \operatorname{gr}_{I_{1}}\left(S_{1}\right) \otimes_{K} \operatorname{gr}_{I_{2}}\left(S_{2}\right) \rightarrow \operatorname{gr}_{I}(S)$ with $f \otimes g \mapsto f g$ is an isomorphism. Here, since $\operatorname{gr}_{I_{1}}\left(S_{1}\right) \rightarrow \operatorname{gr}_{I}(S)$ is injective, we identify $f \in \operatorname{gr}_{I_{1}}\left(S_{1}\right)$ with its image in $\operatorname{gr}_{I}(S)$. A similar identification is made for $g \in \operatorname{gr}_{I_{2}}\left(S_{2}\right)$.

For an ideal $J \subseteq \operatorname{gr}_{I_{i}}\left(S_{i}\right)$ we denote its extension ideal under the canonical (injective) $\operatorname{map} \operatorname{gr}_{I_{i}}\left(S_{i}\right) \rightarrow \operatorname{gr}_{I}(S)$ again by $J$. With this convention we have

Theorem 1.2. Let $I_{1} \subseteq S_{1}=K\left[x_{1}, \ldots, x_{m}\right]$ and $I_{2} \subseteq S_{2}=K\left[y_{1}, \ldots, y_{n}\right]$ be monomial ideals, and let $I=\left(I_{1}, I_{2}\right) S \subseteq S$, where $S=S_{1} \otimes_{K} S_{2}$. Assume that $I_{1}, I_{2}$ and $I$ satisfy the Ratliff condition. Then $\operatorname{Soc}(I)=\operatorname{Soc}\left(I_{1}\right) \operatorname{Soc}\left(I_{2}\right)$.

Proof. Let $A_{i}=\operatorname{gr}_{i_{1}}\left(S_{i}\right)$ for $i=1,2$. Our assumptions imply that $\operatorname{Soc}\left(I_{1}\right)$ is an ideal in $A_{1}, \operatorname{Soc}\left(I_{2}\right)$ an ideal in $A_{2}$ and $\operatorname{Soc}(I)$ an ideal in $A=\operatorname{gr}_{I}(S)$. Then $\operatorname{Soc}\left(I_{1}\right) \otimes_{K} \operatorname{Soc}\left(I_{2}\right)$ is an ideal in $A_{1} \otimes_{K} A_{2}$. We show that $\alpha\left(\operatorname{Soc}\left(I_{1}\right) \otimes_{K} \operatorname{Soc}\left(I_{2}\right)\right)=$ $\operatorname{Soc}(I)$.

The inclusion $\alpha\left(\operatorname{Soc}\left(I_{1}\right) \otimes_{K} \operatorname{Soc}\left(I_{2}\right)\right) \subseteq \operatorname{Soc}(I)$ is obvious. For the converse inclusion, we first notice that $\operatorname{Soc}(I)$ is generated by monomials. Indeed, the elements of degree $k$ in $\operatorname{Soc}(I)$ which are not zero are of the form $u+I^{k+1} \in I^{k} / I^{k+1}$ with $u \in\left(I^{k+1}: \mathfrak{m}\right) \backslash I^{k+1}$, where $\mathfrak{m}=\left(x_{1}, \ldots, x_{m}, y_{1}, \ldots, y_{m}\right)$. Since $I$ is a monomial ideal, $I^{k+1}: \mathfrak{m}$ is a monomial ideal as well, and the assertion follows.

Let $u+I^{k+1} \in \operatorname{Soc}(I)_{k}$ with $u$ a monomial. Since $\alpha$ is a multigraded isomorphism there exists monomials $u_{i} \in S_{i}$ and non-negative positive integers $r$ and $s$ with $r+s=k$ such that $\alpha\left(\left(u_{1}+I_{1}^{r+1}\right) \otimes\left(u_{2}+I_{2}^{s+1}\right)\right)=u+I^{k+1}$. Since $x_{i}\left(u+I^{k}\right)=0$ and since $\alpha$ is an isomorphism, it follows that

$$
\left(x_{i} u_{1}+I_{1}^{r+1}\right) \otimes\left(u_{2}+I_{2}^{s+1}\right)=\left(x_{i} \otimes 1\right)\left(\left(u_{1}+I_{1}^{r+1}\right) \otimes\left(u_{2}+I_{2}^{s+1}\right)\right)=0 .
$$

Note that $u_{2}+I_{2}^{s+1} \neq 0$, because otherwise $\left(u_{1}+I_{1}^{r+1}\right) \otimes\left(u_{2}+I_{2}^{s+1}\right)=0$, and hence $u+I^{k+1}=0$. On the other hand, since $\left(x_{i} u_{1}+I^{r+1}\right) \otimes\left(u_{2}+I^{s+1}\right)=0$, it follows that $\left(x_{i} u_{1}+I_{1}^{r+1}\right)=0$. This is the case for all $i$, which implies that $u_{1}+I_{1}^{r+1} \in \operatorname{Soc}\left(I_{1}\right)$. Similarly we see that $u_{2}+I_{2}^{s+1} \in \operatorname{Soc}\left(I_{2}\right)$.

Let $I \subseteq S$ be a monomial ideal generated in degree $d$, and $u \in S$ a monomial such that $u+I^{m} \in \operatorname{Soc}(I)$ and $u+I^{m} \neq 0$. What can we say about the $\operatorname{degree} \operatorname{deg}(u)$ of $u$ ? Obviously, $\operatorname{deg}(u) \geq m d-1$. If $\operatorname{deg}(u)=m d-1$ for all such elements $u$, then the socle module $\operatorname{Soc}(I)$ has the following good property. 
Proposition 1.3. Let $I \subseteq S$ be a monomial ideal generated in degree d. Suppose that for any positive integer $m$ and for any monomial $u \in\left(I^{m}: \mathfrak{m}\right) \backslash I^{m}$, one has $\operatorname{deg}(u)=m d-1$. Then $\operatorname{Soc}(I)$ is identified with an ideal of $\mathcal{F}(I)$. In particular, $\operatorname{rank} \operatorname{Soc}(I)=1$ if $\operatorname{Soc}(I) \neq 0$.

Proof. Fix a variable $x \in S$. We define $\varphi$ to be the $\operatorname{map} \varphi: \operatorname{Soc}(I) \longrightarrow \mathcal{F}(I)$ with the property that $\varphi\left(u+I^{m}\right)=x u+\mathfrak{m} I^{m}$ for any $u+I^{m} \in\left(I^{m}: \mathfrak{m}\right) / I^{m}$ and that $\varphi\left(u_{1}+u_{2}\right)=\varphi\left(u_{1}\right)+\varphi\left(u_{2}\right)$ for any $u_{1}, u_{2} \in \operatorname{Soc}(I)$.

It is clear that $\varphi$ is well-defined. Note that if a monomial $u \in\left(I^{m}: \mathfrak{m}\right) \backslash I^{m}$ then $\operatorname{deg}(x u)=m d$ and $x u \notin \mathfrak{m} I^{m}$, we have $\varphi$ is injective. It remains to show that $\varphi$ is a $\mathcal{F}(I)$-module homomorphism. Let $u+I^{m} \in\left(I^{m}: \mathfrak{m}\right) / I^{m}$ and $v+\mathfrak{m} I^{r} \in I^{r} / \mathfrak{m} I^{r}$. Then

$$
\varphi\left(\left(v+\mathfrak{m} I^{r}\right)\left(u+I^{m}\right)\right)=\varphi\left(v u+I^{m+r}\right)=x v u+\mathfrak{m} I^{m+r}=\left(v+\mathfrak{m} I^{r}\right) \varphi\left(u+I^{m}\right) .
$$

By this equality and since $\varphi$ preserves the addition, it follows that $\varphi$ is indeed a $\mathcal{F}(I)$-module homomorphism, as required.

Finally, we note that $\mathcal{F}(I)$ is an integral domain (in fact, it is a subalgebra of the polynomial ring $S$ ) since $I$ is generated in a single degree. Now, the last result follows since every nonzero ideal of an integral domain has rank 1.

Unfortunately, the inequality $\operatorname{deg}(u) \geq m d-1$, where $u$ is a homogeneous element in $\left(I^{m}: \mathfrak{m}\right) \backslash I^{m}$, can be strict. Indeed, consider the edge ideal $I(G)$ of the graph $G$ consisting of two disjoint 3-cycles which are connected by a path of length 2 , see $G_{1}$ in Figure 1. Then

$$
I(G)=\left(x_{1} x_{2}, x_{1} x_{3}, x_{2} x_{3}, y_{1} y_{2}, y_{2} y_{3}, y_{2} y_{3}, x_{1} z, y_{1} z\right) .
$$

It can be easily seen that $u \in\left(I(G)^{3}: \mathfrak{m}\right) \backslash I(G)^{3}$, where $u=x_{1} x_{2} x_{3} y_{1} y_{2} y_{3}$. Here, $\operatorname{deg}(u)>3 \cdot 2-1$.

On the other hand, we have

Proposition 1.4. Suppose that the graded ideal $I$ in the polynomial ring $S$ has $d$-linear resolution. Then each homogeneous element in $(I: \mathfrak{m}) \backslash I$ is of degree $d-1$

Proof. Let

$$
\mathbb{F}: 0 \rightarrow F_{n-1} \rightarrow \cdots \rightarrow F_{1} \rightarrow F_{0} \rightarrow I \rightarrow 0 .
$$

be the graded free $S$-resolution of $I$. Since $I$ has $d$-linear resolution, it follows that $F_{n-1}=S(-d-(n-1))^{\beta_{n-1}}$.

We have the following isomorphisms of graded modules

$$
K(-d-(n-1))^{\beta_{n-1}} \cong \operatorname{Tor}_{n-1}(K, I) \cong \operatorname{Tor}_{n}(K, S / I) \cong H_{n}\left(x_{1}, \ldots, x_{n} ; S / I\right) .
$$

Here $H_{n}\left(x_{1}, \ldots, x_{n} ; S / I\right)$ denotes the $n$th Koszul homology of $S / I$ with respect to the sequence $x_{1}, \ldots, x_{n}$. Note that $H_{n}\left(x_{1}, \ldots, x_{n} ; S / I\right)=((I: \mathfrak{m}) / I) \bigwedge^{n} E$, where $E=\bigoplus_{i=1}^{n}(S / I) e_{i}$. Hence $((I: \mathfrak{m}) / I) \bigwedge^{n} E=((I: \mathfrak{m}) / I)\left(e_{1} \wedge e_{2} \wedge \cdots \wedge e_{n}\right)=$ $K(-d-(n-1))^{\beta_{n-1}}$. Comparing degrees we obtain the desired conclusion, since $\operatorname{deg}\left(e_{1} \wedge e_{2} \wedge \cdots \wedge e_{n}\right)=n$. 
Proposition 1.4 implies that if $I$ is a graded ideal generated in degree $d$ which has the property that all its powers have linear resolution, then $\operatorname{Soc}(I)$ is generated by elements of the form $f+I^{k}$ where $f$ is homogeneous of degree $k d-1$. Classes of such ideals are the polymatroidal ideals which we consider in the last section of this paper. In Proposition 2.2, we provide a large class of edge ideals $I$ which may have not linear resolutions, but the equality $\operatorname{deg}(u)=m d-1=2 m-1$ still holds for any monomial $u \in\left(I^{m}: \mathfrak{m}\right) \backslash I^{m}$.

There is a natural condition on monomial ideals $I$ generated in degree $d$ which guarantees the existence of at least one generator $u+I^{k}$ of $\operatorname{Soc}(I)$ with $\operatorname{deg} u=k d-1$.

Let $G(I)=\left\{u_{1}, \ldots, u_{m}\right\}$. The linear relation graph $\Gamma$ of $I$ is the graph with edge set

$$
E(\Gamma)=\left\{\{i, j\}: \text { there exist } u_{k}, u_{l} \in G(I) \text { such that } x_{i} u_{k}=x_{j} u_{l}\right\}
$$

and vertex set $V(\Gamma)=\bigcup_{\{i, j\} \in E(\Gamma)}\{i, j\}$.

In the special case that $I=I(G)$ is the edge ideal of a simple graph $G$ on the vertex set $[n]$, the linear relation graph $\Gamma$ of $I(G)$ has edge set

$$
\{\{i, j\}: i, j \in V(G) \text { and } i \text { and } j \text { have a common neighbor in } G\} .
$$

The next result follows from [8, Corollary 3.4].

Proposition 1.5. Let $I \subseteq S$ be a monomial ideal generated in degree $d$ with linear relation graph $\Gamma$. Suppose that $\Gamma$ has $n$ vertices and that $\Gamma$ is connected. Then there exists a monomial $u \in\left(I^{n}: \mathfrak{m}\right) \backslash I^{n}$ of degree $d n-1$. In particular, $\operatorname{Soc}(I)$ admits a generator $u+I^{k}$ with $k \leq n$ and $\operatorname{deg} u=k d-1$.

\section{EDGE IDEALS}

In this section, we always assume that $G$ is a simple graph on vertex set $V(G)=$ $\left\{x_{1}, \ldots, x_{n}\right\}$ unless otherwise stated. The set of edges of $G$ is denoted by $E(G)$. Let $S=K\left[x_{1}, \ldots, x_{n}\right]$ be the polynomial ring over a field $K$. The edge ideal of $G$, denoted by $I(G)$, is the ideal of $S$ generated by all square-free monomials $x_{i} x_{j}$ such that $\left\{x_{i}, x_{j}\right\} \in E(G)$. We often write $x_{i} x_{j} \in E(G)$ instead of $\left\{x_{i}, x_{j}\right\} \in E(G)$. By abusing notation, we use $x_{i} x_{j}$ to refer to both the edge $x_{i} x_{j} \in E(G)$ and the monomial $x_{i} x_{j} \in I(G)$. We will study the socle module of an edge ideal of $G$. In view of Theorem 1.2, we always assume that $G$ is a connected graph.

Recall some necessary graphic notions. A walk in $G$ is an alternating sequence of vertices and edges $x_{1}, e_{1}, x_{2}, e_{2}, \ldots, e_{t-1}, x_{t}$ in which $e_{i}=x_{i} x_{i+1}$. The number $t-1$ is the length of this walk. We often write this walk simply as: $x_{1}-x_{2}-\cdots-x_{t}$. If $x_{1}=x_{t}$, it is called a closed walk. A path is a simple walk, that is, a walk in which each vertex appears exactly once. A cycle is a closed walk in which each vertex appears exactly once except the first and the last vertices. A cycle of odd length is called an odd cycle.

We use $\{\{\ldots\}\}$ to refer to a multiset. For examples, $\{\{1,2\}\} \neq\{\{1,2,2\}\}$, $\{\{1,2,2\}\} \subseteq\{\{1,2,2,3,4\}\}$, but $\{\{1,2,2\}\} \nsubseteq\{\{1,2,3,4\}\}$, and $\{\{1,2,2,3,3\}\} \cap$ $\{\{2,2,2,3\}\}=\{\{2,2,3\}\}$. The next lemma, which is a variation of [2, Theorem 6.1 and Theorem 6.7], plays a key role in this section. 
Lemma 2.1. Let $G$ be a simple graph with edge ideal $I=I(G)$. Let $m \geq 1$ be an integer and let $e_{1}, e_{2}, \ldots, e_{m}$ (maybe repeatedly) be edges of $G$ and $\mathbf{v} \in S$ a monomial such that $e_{1} e_{2} \cdots e_{m} \mathbf{v} \in I^{m+1}$. Then there exist variables $w$ and $y$ with $w y \mid \mathbf{v}$ and an odd walk in $G$ connecting $w$ to $y$ :

$$
w=z_{1}-z_{2}-z_{3}-\cdots-z_{2 t}-z_{2 t+1}-z_{2 t+2}=y
$$

such that $\left\{\left\{z_{2} z_{3}, \ldots, z_{2 t} z_{2 t+1}\right\}\right\} \subseteq\left\{\left\{e_{1}, \ldots, e_{m}\right\}\right\}$. Here, if $t=0$, then the walk means the edge $w-y$ (i.e., the edge wy).

Let $U, W$ be two subsets of $V(G)$. Then the distance between $U$ and $W$, denoted by $\operatorname{dist}(U, W)$, is defined as

$$
\operatorname{dist}(U, W)=\min \{\operatorname{dist}(x, y): x \in U, y \in W\},
$$

where $\operatorname{dist}(x, y)$ denotes the minimal of lengths of the paths between $x$ and $y$. By convention, $\operatorname{dist}(x, y)=0$ if $x=y$ and $\operatorname{dist}(x, y)=\infty$ if there is no path between $x$ and $y$. We observe the following easy fact: every odd closed walk (i.e., closed walk of odd length) contains an odd cycle. More precisely, if $W$ is a closed odd walk of $G$, then $W$ has a subgraph $C$ such that $C$ is an odd cycle. Here, $C$ is a subgraph of $W$ means $V(C) \subseteq V(W)$ and $E(C) \subseteq E(W)$.

Proposition 2.2. Let $G$ be a simple connected graph such that $\operatorname{dist}\left(V\left(C_{1}\right), V\left(C_{2}\right)\right) \leq$ 1 for any two odd cycles $C_{1}, C_{2}$ of $G$. Then for any positive integer $m$ and for any monomial $\mathbf{u}$ with $\mathbf{u} \in\left(I^{m}: \mathfrak{m}\right) \backslash I^{m}$, one has $\operatorname{deg}(\mathbf{u})=2 m-1$ and $\mathbf{u}$ can be written as $\mathbf{u}=x_{1} x_{2} \cdots x_{2 k+1} e_{1} \cdots e_{m-k-1}$, where $e_{i}$ is an edge for each $i$ and $x_{1}-x_{2}-\cdots-x_{2 k+1}-x_{1}$ is an odd cycle.

Proof. Let $\mathbf{u}$ be a monomial in $\left(I^{m}: \mathfrak{m}\right) \backslash I^{m}$. Then $\mathbf{u}=e_{1} \cdots e_{m-1} \mathbf{v}$, where $e_{i} \in E(G)$ for $i=1, \ldots, m-1$ and $\mathbf{v}$ is a monomial. We need to $\operatorname{show} \operatorname{deg}(\mathbf{v})=1$. Assume on the contrary that there are variables $x, y$ such that $x y$ divides $\mathbf{v}$. Since $x \mathbf{u} \in I^{m}$, it follows from Lemma 2.1 that there is an integer $t \geq 1$ and a walk

$$
z_{1}-z_{2}-z_{3}-\cdots-z_{2 t}-z_{2 t+1}-z_{2 t+2}
$$

in $G$ such that $z_{1} z_{2 t+2}$ divides $x \mathbf{v}$ and

$$
\left\{\left\{z_{2} z_{3}, z_{4} z_{5}, \ldots, z_{2 t} z_{2 t+1}\right\}\right\} \subseteq\left\{\left\{e_{1}, \ldots, e_{m-1}\right\}\right\} .
$$

Since

$$
z_{1}\left(z_{2} z_{3}\right) \cdots\left(z_{2 t} z_{2 t+1}\right) z_{2 t+2}=\left(z_{1} z_{2}\right)\left(z_{3} z_{4}\right) \cdots\left(z_{2 t+1} z_{2 t+2}\right) \in I^{t+1}
$$

we have

$$
z_{1} z_{2 t+2} e_{1} \cdots e_{m-1} \in I^{m} .
$$

This implies that $z_{1} z_{2 t+2}$ does not divide $\mathbf{v}$, since $\mathbf{u} \notin I^{m}$. Hence, we have

$$
z_{1} z_{2 t+2}\left|x \mathbf{v}, \quad z_{1} z_{2 t+2} \nmid \mathbf{v}, \quad x y\right| \mathbf{v} .
$$

From this it follows that $z_{1}=z_{2 t+2}=x$ and $x \neq y$.

By the argument above, we see that $x=z_{1}-z_{2}-z_{3}-\cdots-z_{2 t}-z_{2 t+1}-z_{2 t+2}=x$ is an odd closed walk. Hence, by the relabelling of vertices, there exist odd closed 
walks $C_{1}: x=x_{1}-x_{2}-x_{3}-\cdots-x_{2 k}-x_{2 k+1}-x_{1}=x$ and $C_{2}: y=y_{1}-y_{2}-\cdots-$ $y_{2 \ell}-y_{2 \ell+1}-y_{1}=y$ such that

$$
\left\{\left\{f_{1}, \ldots, f_{k}\right\}\right\} \subseteq\left\{\left\{e_{1}, \ldots, e_{m-1}\right\}\right\}
$$

and

$$
\left\{\left\{g_{1}, \ldots, g_{\ell}\right\}\right\} \subseteq\left\{\left\{e_{1}, \ldots, e_{m-1}\right\}\right\},
$$

where $f_{i}=x_{2 i} x_{2 i+1}$ for $i=1, \ldots, k$ and $g_{i}=y_{2 i} y_{2 i+1}$ for $i=1, \ldots, \ell$.

Denote by $F$ the multi-set $\left\{\left\{f_{1}, \ldots, f_{k}\right\}\right\} \cap\left\{\left\{g_{1}, \ldots, g_{\ell}\right\}\right\}$. We firstly assume that $F \neq \emptyset$. Then

$$
\mathbf{u}_{1}:=\frac{x y f_{1} \cdots f_{k} g_{1} \cdots g_{\ell}}{\prod_{e \in F} e} \text { divides } \quad \mathbf{u}
$$

and moreover,

This implies

$$
\frac{\mathbf{u}}{\mathbf{u}_{1}} \text { is the product of some edges . }
$$

$$
\mathbf{u}_{1} \in I^{k+\ell-|F|} \backslash I^{k+\ell-|F|+1} .
$$

Let $s$ be the minimal of $i$ with $f_{i} \in F$ and let $1 \leq t \leq \ell$ such that $f_{s}=g_{t}$. Then either $x_{2 s}=y_{2 t+1}$ and $x_{2 s+1}=y_{2 t}$ or $x_{2 s}=y_{2 t}$ and $x_{2 s+1}=y_{2 t+1}$. Suppose that $x_{2 s}=y_{2 t+1}$ and $x_{2 s+1}=y_{2 t}$. In this case, $\mathbf{u}_{1}$ could be written as

$$
\mathbf{u}_{1}=\prod_{i=0}^{s-1}\left(x_{2 i+1} x_{2 i+2}\right) \cdot\left(x_{2 s+1} y_{2 t-1}\right) \cdot \prod_{i=1}^{t-1}\left(y_{2 i-1} y_{2 i}\right) \cdot \prod_{i=t+1}^{\ell}\left(y_{2 i} y_{2 i+1}\right) \cdot \frac{\prod_{i=s+1}^{k} f_{i}}{\prod_{e \in F \backslash\left\{\left\{f_{s}\right\}\right\}} e} .
$$

By the choice of $s$, we see that $F \backslash\left\{\left\{f_{s}\right\}\right\} \subseteq\left\{\left\{f_{s+1}, \cdots, f_{k}\right\}\right\}$. From this it follows that $\mathbf{u}_{1}$ is the product of edges and $\mathbf{u}_{1} \in I^{k+\ell-|F|+1}$. This is a contradiction. The case that $x_{2 s}=y_{2 t}$ and $x_{2 s}=y_{2 t+1}$ yields a contradiction similarly.

Secondly, we assume that $F=\emptyset$. Then

$$
\mathbf{u}_{2}:=x f_{1} \cdots f_{k} y g_{1} \cdots g_{\ell}=x_{1} x_{2} \cdots x_{2 k+1} y_{1} y_{2} \cdots y_{2 \ell+1} \in I^{k+\ell} \backslash I^{k+\ell+1} .
$$

If $V\left(C_{1}\right) \cap V\left(C_{2}\right)=\emptyset$, there exist some vertex of $C_{1}$, say $x_{1}$, and some vertex of $C_{2}$, say $y_{1}$, such that $x_{1} y_{1} \in E(G)$, since $\operatorname{dist}\left(V\left(C_{1}\right), V\left(C_{2}\right)\right) \leq 1$ (Here, we use the observation that every odd closed walk contains an odd cycle). Thus,

$$
\mathbf{u}_{2}=\left(x_{1} y_{1}\right)\left(x_{2} x_{3}\right) \cdots\left(x_{2 k} x_{2 k+1}\right)\left(y_{2} y_{3}\right) \cdots\left(y_{2 k} y_{2 k+1}\right) \in I^{k+\ell+1},
$$

a contradiction. If $V\left(C_{1}\right) \cap V\left(C_{2}\right) \neq \emptyset$, we may harmlessly assume that $x_{1}=y_{1}$. Then

$$
\mathbf{u}_{2}=\left(x_{1} y_{2 \ell+1}\right)\left(x_{2} x_{3}\right) \cdots\left(x_{2 k} x_{2 k+1}\right)\left(y_{1} y_{2}\right) \cdots\left(y_{2 k-1} y_{2 k}\right) \in I^{k+\ell+1} .
$$

This is also a contradiction. The contradictions obtained above show that $\operatorname{deg}(\mathbf{v})=$ 1 and $\operatorname{deg}(\mathbf{u})=2 m-1$. Moreover, by the first paragraph of this proof, we may write $\mathbf{u}$ as $\mathbf{u}=x_{1} x_{2} \cdots x_{2 k+1} e_{1} \cdots e_{m-k-1}$, where $\left\{x_{1}, \ldots, x_{2 k+1}\right\}$ are the vertex set of an odd cycle and $e_{i} \in E(G)$ for $i=1, \cdots, m-k-1$. Here, we use the fact that if $z_{1}-z_{2}-\cdots-z_{2 k+1}-z_{1}$ is an odd closed walk containing an odd cycle $x_{1}-x_{2}-\cdots-x_{2 \ell+1}-x_{1}$ then $z_{1} \cdots z_{2 k+1}$ is the product of $x_{1} \cdots x_{2 \ell+1}$ and some edges. 
Example 2.3. Let $G_{1}, G_{2}$ be in Figure 1, and set $I_{i}=I\left(G_{i}\right)$ for $i=1,2$. Then $x_{1} x_{2} x_{3} x_{4} x_{5} x_{6} \in\left(I_{1}^{3}: \mathfrak{m}\right) \backslash I_{1}^{3}$. This illustrates that the requirement on the distance of odd cycles in Proposition 2.2 cannot be removed.

In contrast, if a monomial $\mathbf{u} \in\left(I_{2}^{m}: \mathfrak{m}\right) \backslash I_{2}^{m}$, then $\operatorname{deg}(\mathbf{u})=2 m-1$. Thus, the converse of Proposition 2.2 does not hold.

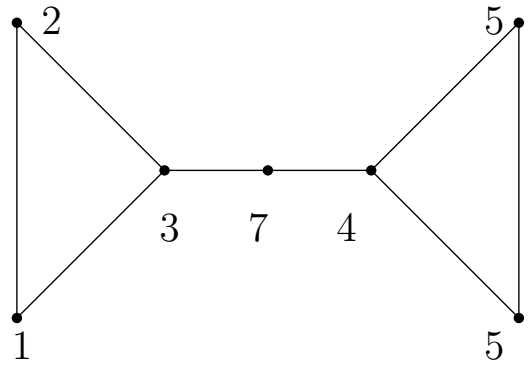

$G_{1}$

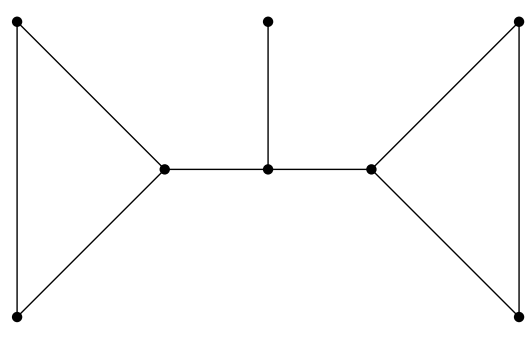

$G_{2}$

FigURE 1.

Combining Proposition 1.3 with Proposition 2.2, we obtain the following result.

Corollary 2.4. Under the same assumptions on $G$ as in Proposition 2.2, the socle module $\operatorname{Soc}(I(G))$ is identified with an ideal of $\mathcal{F}(I)$.

We now come to the main result of this section. If $G$ is a unicyclic graph with the unique odd cycle $C_{2 k+1}$, we use the notion $E^{*}(G)$ to stand for the following subset of $E(G)$ :

$$
E^{*}(G)=\left\{e \in E(G) \backslash E\left(C_{2 k+1}\right): \quad e \text { is not a leaf of } G\right\} .
$$

Here, an edge $e=y_{1} y_{2} \in E(G)$ is called a leaf of $G$ if either $y_{1}$ or $y_{2}$ is adjacent to only one vertex of $G$.

Proposition 2.5. Let $G$ be a unicyclic graph containing an odd cycle $C_{2 k+1}$ with $V\left(C_{2 k+1}\right)=\left\{x_{1}, x_{2}, \ldots, x_{2 k+1}\right\}$. Let $u \in S$ a monomial and $m$ a positive integer. Then

$$
\mathbf{u} \in\left(I^{m}: \mathfrak{m}\right) \backslash I^{m} \text { if and only if } \mathbf{u}=\left(x_{1} x_{2} \cdots x_{2 k+1}\right) \prod_{e \in E_{1}} e^{m_{e}},
$$

where $m_{e} \geq 1$ for all $e \in E_{1}, E^{*}(G) \subseteq E_{1}$, and $\sum_{e \in E_{1}} m_{e}=m-k-1$.

Proof. Sufficiecy.

Note that $\operatorname{deg}(\mathbf{u})=2 k+1+2(m-k-1)=2 m-1$, it is clear that $\mathbf{u} \notin I^{m}$. We only need to show $y \mathbf{u} \in I^{m}$ for any $y \in V(G)$. Let $y \in V(G)$.

Case (i): $\operatorname{dist}\left(y, V\left(C_{2 k+1}\right)\right)=0$, that is, $y \in V\left(C_{2 k+1}\right)$. We may harmlessly assume that $y=x_{1}$. Then

$$
\left.\mathbf{u} y=\left(x_{1} x_{2} \cdots x_{2 k+1}\right)\left(\prod_{e \in E_{1}} e^{m_{e}}\right) x_{1}=\left[\left(x_{1} x_{2}\right)\left(x_{3} x_{4}\right) \cdots\left(x_{2 k+1} x_{1}\right)\right)\right] \cdot \prod_{e \in E_{1}} e^{m_{e}} \in I^{m} .
$$


Case (ii): $\operatorname{dist}\left(y, C_{2 k+1}\right)>0$. Then there exits $x \in V\left(C_{2 k+1}\right)$, say $x=x_{1}$, such that $\operatorname{dist}\left(y, V\left(C_{2 k+1}\right)\right)=\operatorname{dist}(x, y)$. Write the unique path between $y$ and $x_{1}$ as:

$$
y=y_{0}-y_{1}-y_{2}-\cdots-y_{\ell}-x_{1} .
$$

Suppose that $\ell=2 k$ for some $k \geq 1$. Then, since $y_{1} y_{2}, y_{3} y_{4}, \ldots, y_{2 k-1} y_{2 k}$ are pairwise different edges belonging to $E^{*}(G)$, we may write $y \mathbf{u}$ as:

$$
y \mathbf{u}=\left(x_{1} \cdots x_{2 k+1}\right) y_{0}\left(y_{1} y_{2}\right) \cdots\left(y_{2 k-1} y_{2 k}\right) \mathbf{v},
$$

where $\mathbf{v}$ is the product of some edges. It follows that

$$
y \mathbf{u}=\left(y_{0} y_{1}\right) \cdots\left(y_{2 k-2} y_{2 k-1}\right)\left(y_{2 k} x_{1}\right)\left(x_{2} x_{3}\right) \cdots\left(x_{2 k} x_{2 k+1}\right) \mathbf{v}
$$

and $y \mathbf{u}$ is also the product of edges. Since $\operatorname{deg}(y \mathbf{u})=2 m$, we have $y \mathbf{u} \in I^{m}$. Suppose next that $\ell=2 k+1$. Then we may write $y \mathbf{u}$ as

$$
y \mathbf{u}=\left(x_{1} \cdots x_{2 k+1}\right) y_{0}\left(y_{1} y_{2}\right) \cdots\left(y_{2 k-1} y_{2 k}\right)\left(y_{2 k+1} x_{1}\right) \mathbf{v}
$$

such that $\mathbf{v}$ is the product of edges. Thus,

$$
y \mathbf{u}=\left(y_{0} y_{1}\right) \cdots\left(y_{2 k} y_{2 k+1}\right)\left(x_{1} x_{2}\right) \cdots\left(x_{2 k-1} x_{2 k}\right)\left(x_{1} x_{2 k+1}\right) \mathbf{v}
$$

and it is also the product of edges. Note that $\operatorname{deg}(y \mathbf{u})=2 m$, we have $y \mathbf{u} \in I^{m}$.

Necessity.

Let $\mathbf{u} \in\left(I^{m}: \mathfrak{m}\right) \backslash I^{m}$ be a monomial. Then, according to Proposition 2.2 , there exists a subset $E_{1} \subseteq E(G)$ such that

$$
\mathbf{u}=\left(x_{1} \cdots x_{2 k+1}\right) \prod_{e \in E_{1}} e^{m_{e}}
$$

where $m_{e} \geq 1$ for any $e \in E_{1}$ and $\sum_{e \in E_{1}} m_{e}=m-k-1$. We only need to prove $E^{*}(G) \subseteq E_{1}$.

Let $e=y_{1} y_{2} \in E^{*}(G)$. There exits a unique vertex in $C_{2 k+1}$, say $x_{1}$, such that $\operatorname{dist}\left(x_{1}, y_{i}\right)=\operatorname{dist}\left(V\left(C_{2 k+1}\right), y_{i}\right)$ for $i=1,2$. Moreover, we may harmlessly assume that $\operatorname{dist}\left(y_{2}, x_{1}\right)=\operatorname{dist}\left(y_{1}, x_{1}\right)+1$. It is clear that $y_{2} \notin\left\{x_{1}, \ldots, x_{2 k+1}\right\}$. Since $y_{1} y_{2}$ is not a leaf, there exists a vertex $z$ of $G$ such that $\operatorname{dist}\left(z, x_{1}\right)=\operatorname{dist}\left(y_{2}, x_{1}\right)+1$ and $z y_{2} \in E(G)$.

Since $z \mathbf{u}=x_{1} z\left(x_{2} x_{3}\right) \cdots\left(x_{2 k} x_{2 k+1}\right) \prod_{e \in E_{1}} e^{m_{e}} \in I^{m}$, it follows from Lemma 2.1 that there exists an odd walk connecting $z$ to $x_{1}$ :

$$
z=z_{1}-z_{2}-z_{3}-\cdots-z_{2 t}-z_{2 t+1}-z_{2 t+2}=x_{1}
$$

such that

$$
\left\{\left\{z_{2} z_{3}, \ldots, z_{2 t} z_{2 t+1}\right\}\right\} \subseteq\left\{\left\{m_{e} e: e \in E_{1}, x_{2} x_{3}, \ldots, x_{2 k} x_{2 k+1}\right\}\right\},
$$

where $m_{e} e$ means that $e$ appears $m_{e}$ times. We may assume that $W$ has the shortest length among such walks.

Let $\ell$ be the minimal of numbers $\{1,2, \ldots, 2 t+2\}$ such that $z_{\ell}=x_{1}$. Then, any subset of $\left\{z_{1}, \ldots, z_{\ell-1}\right\}$ cannot form any odd closed walk in $G$, since $\left\{x_{1}, \ldots, x_{2 k-1}\right\} \cap$ $\left\{z_{1}, \ldots, z_{\ell-1}\right\}=\emptyset$. We claim that $z \notin\left\{z_{2}, \ldots, z_{\ell-1}\right\}$. It is clear that $z \neq z_{2}$. If $z=z_{2 s}$ for some $s$ with $4 \leq 2 s \leq \ell-1$, then $z=z_{1}-z_{2}-\cdots-z_{2 s}=z$ is an odd closed walk, which is impossible. If $z=z_{2 s+1}$ for some $s>0$ with $2 s+1 \leq \ell-1$, then $z=z_{2 s+1}-z_{2 s+2}-\cdots-z_{2 t}-z_{2 t+1}-z_{2 t+2}=x_{1}$ is a shorter 
walk than (W), a contradiction. Thus, $z \notin\left\{z_{2}, \ldots, z_{\ell-1}\right\}$, as claimed. From this it follows that $\operatorname{dist}\left(z_{2}, x_{1}\right)=\operatorname{dist}\left(z_{1}, x_{1}\right)-1$ and $z_{2}=y_{2}$. In a similar way, we have $y_{2} \notin\left\{z_{3}, \ldots, z_{\ell-1}\right\}$ and $z_{3}=y_{1}$. Hence, $e \in E_{1}$ by (II).

In the following, if $G$ is a unicyclic graph with odd cycle $x_{1}-x_{2}-\cdots-x_{2 k+1}-x_{1}$, we use $d_{G}$ to denote the number

$$
d_{G}=\left|E^{*}(G)\right|+k+1
$$

and use $\mathbf{u}_{G}$ to denote the monomial

$$
\mathbf{u}_{G}=\left(x_{1} \cdots x_{2 k+1}\right) \prod_{e \in E^{*}(G)} e .
$$

It is clear from Proposition 2.5 that $\mathbf{u}_{G}+I^{d_{G}}$ is the unique minimal generator of $\operatorname{Soc}(I(G))$.

Theorem 2.6. Let $G$ be a unicyclic nonbipartite graph. Then $\operatorname{Soc}(I(G))$ is a free $\mathcal{F}(I(G))$-module of rank 1. More precisely,

$$
\operatorname{Soc}(I(G)) \cong \mathcal{F}(I(G))\left(-d_{G}+1\right) .
$$

Proof. Denote $I(G)$ by $I$. In view of Proposition 2.5, we have

$$
\operatorname{Soc}(I)=\left(\mathbf{u}_{G}+I^{d_{G}}\right) \mathcal{F}(I)
$$

and so it is a cyclic $\mathcal{F}(I)$-module. According to Proposition 2.4, $\mathbf{u}_{G}+I^{d_{G}}$ has no non-zero annihilator in $\mathcal{F}(I)$, namely, $f\left(\mathbf{u}_{G}+I^{d_{G}}\right) \neq 0$ for any $0 \neq f \in \mathcal{F}(I)$. Thus, $\operatorname{Soc}(I)$ is a free $\mathcal{F}(I)$-module of rank 1 . The last isomorphism follows since $\mathbf{u}_{G}+I^{d_{G}} \in \operatorname{Soc}(I)$ is of degree $d_{G}-1$.

The converse of Theorem 2.6 is not true as shown by the following example.

Example 2.7. Let $G$ be the graph obtained by adding an edge $x_{1} x_{3}$ to the 5 -cycle $x_{1}-x_{2}-\cdots-x_{5}-x_{1}$. Then $x_{1} x_{2} x_{3}+I(G)^{2}$ is the unique minimal generator of $\operatorname{Soc}(I(G))$ and so $\operatorname{Soc}(I(G))$ is also a free $\mathcal{F}(I(G))$-module of rank 1 .

In the final part of this section, we give an application of our results to the depth stability index of an edge ideal. Let $I$ be an ideal of $S$. Recall that the stability index of $I$, denoted by $\operatorname{dsatb}(I)$, is defined to be

$$
\operatorname{dsatb}(I)=\min \left\{t \geq 0: \operatorname{depth}\left(I^{t}\right)=\operatorname{depth}\left(I^{t+i}\right) \text { for all } i \geq 0\right\} .
$$

Let $G$ be any simple connected graph. By a spanning subgraph of $G$, we mean a connected subgraph of $G$ with the same vertex set as $G$. Thus, a spanning unicyclic nonbipartite subgraph of $G$ means a spanning subgraph of $G$ which is unicyclic and nonbipartite. Assume now $H$ is a spanning unicyclic nonbipartite subgraph of $G$. It is easy to check that $\mathbf{u}_{H} \in\left(I(G)^{d_{H}}: \mathfrak{m}\right) \backslash I(G)^{d_{H}}$ and $\mathbf{u}_{H}+I(G)^{d_{H}}$ is a nonzero homogeneous element of degree $d_{H}-1$ in $\operatorname{Soc}(I(G))$. Note that $\operatorname{dstab}(I(G))=$ $\min \left\{i+1: \operatorname{Soc}(I(G))_{i} \neq 0\right\}$, the following result follows.

Corollary 2.8. Let $G$ be a simple connected nonbipartite graph. Then $\operatorname{dstab}(I(G)) \leq \min \left\{d_{H}: H\right.$ is a spanning unicyclic nonbipartite subgraph of $\left.G\right\}$. 
Let $H$ be a spanning unicyclic nonbipartite subgraph of $G$ such that the unique cycle of $H$ has length $2 k-1$, where $2 k-1$ be the maximal length of odd cycles of $G$. Then

$$
d_{H}=|V(H)|-\varepsilon_{0}(H)-k+1 \leq|V(G)|-\varepsilon_{0}(G)-k+1,
$$

where $\varepsilon_{0}(\bullet)$ is the number of leaves of a simple graph $\bullet$. From this fact, we see that Corollary 2.8 implies [16, Proposition 2.4], which says $\operatorname{dstab}(I(G)) \leq|V(G)|-$ $\varepsilon_{0}(G)-k+1$, if $G$ is a connected nonbipartite graph.

Example 2.9. Let $G$ be the complete graph with $2 k-1$ vertices, where $k \geq 2$. According to Corollary 2.8, we see that $\operatorname{dstab}(I(G)) \leq 2$ and $\operatorname{so} \operatorname{dstab}(I(G))=2$. However, by [16, Proposition 2.4], we can only obtain $\operatorname{dstab}(I(G)) \leq k$.

The following example illustrates the inequality in Corollary 2.8 may be strict.

Example 2.10. Let $G$ be the graph depict in Figure 2 and $I$ the edge ideal of $G$. Then $\operatorname{Soc}(I)$ is minimally generated by $x_{1} x_{2} x_{3} x_{4} x_{5}+I^{3}$ and $x_{1} x_{2} x_{3}^{3} x_{4} x_{5}+I^{4}$ by CoCoA [1. This implies $\operatorname{dstab}(I)=3$. Let $H_{1}=G \backslash\left\{x_{1} x_{2}\right\}, H_{2}=G \backslash\left\{x_{4} x_{5}\right\}$, $H_{3}=G \backslash\left\{x_{3} x_{1}\right\}, H_{4}=G \backslash\left\{x_{3} x_{2}\right\}, H_{5}=G \backslash\left\{x_{3} x_{4}\right\}$, and $H_{6}=G \backslash\left\{x_{3} x_{5}\right\}$. Then $H_{1}, \ldots, H_{6}$ are all the spanning unicyclic nonbipartite subgraphs of $G$. However, $d_{H_{i}}=4$ for $i=1, \ldots, 6$.

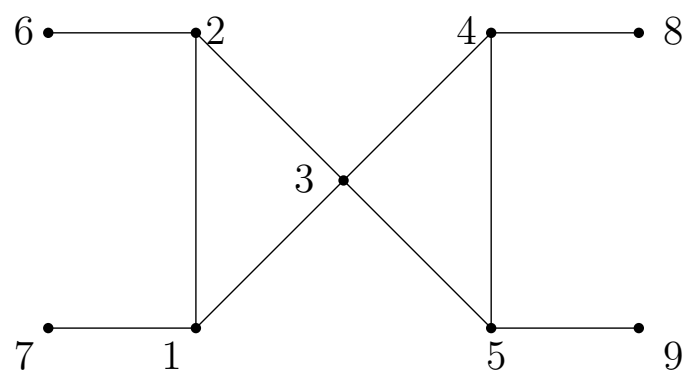

FiguRE 2.

\section{Polymatroidal Ideals}

In this section we want to investigate the socle modules $\operatorname{Soc}^{*}(I)$ of a polymatroidal ideal. We refer to [6] or [7, Chapter 12] for the definition and basic properties of polymatroidal ideals.

Let $I$ be a polymatroidal ideal generated in degree $d$, then $I$ has a linear resolution. Recall that $\operatorname{soc}(I)$ is the monomial ideal generated in degree $d-1$ such that $(I$ : $\mathfrak{m})=I+\operatorname{soc}(I)$. The first basic question in our context is

"is $\operatorname{soc}(I)$ always a polymatroial ideal if I is a polymatroidal ideal?"

We cannot answer this question in its full generality. In what follows we confine our research to a special type of polymatroidal ideals - PLP-polymatroidal ideals, which were introduced firstly in [15] and were redefined and studied further in [11]. 
Denote by $G(I)$ the unique minimal set of generators of a monomial ideal $I$. Let $\mathbf{a}=\left(a_{1}, \ldots, a_{n}\right), \mathbf{b}=\left(b_{1}, \ldots, b_{n}\right), \boldsymbol{\alpha}=\left(\alpha_{1}, \ldots, \alpha_{n}\right), \boldsymbol{\beta}=\left(\beta_{1}, \ldots, \beta_{n}\right)$ be vectors in $\mathbb{Z}_{+}^{n}$. Assume that $\alpha_{1} \leq \alpha_{2} \leq \cdots \leq \alpha_{n}=d, \beta_{1} \leq \beta_{2} \leq \cdots \leq \beta_{n}=d$ and $a_{i} \leq b_{i}$ and $\alpha_{i} \leq \beta_{i}$ for $i=1, \ldots, n$. Here, $d$ is a given integer. We also assume that $\alpha_{1}=a_{1}$ and $\beta_{1}=b_{1}$. Recall from [11] that the PLP-polymatroidal ideal $I$ of type $(\mathbf{a}, \mathbf{b} \mid \boldsymbol{\alpha}, \boldsymbol{\beta})$ is the polymatroidal ideal in $K\left[x_{1}, \ldots, x_{n}\right]$ generated by $\mathbf{x}^{\mathbf{u}}$ with $\mathbf{u}=\left(u_{1}, \ldots, u_{n}\right)$ satisfying

$$
\begin{aligned}
a_{i} \leq u_{i} \leq b_{i}, & \text { for } \quad i=1, \ldots, n \\
\alpha_{i} \leq u_{1}+\cdots+u_{i} \leq \beta_{i}, & \text { for } \quad i=1, \ldots, n
\end{aligned}
$$

The PLP-polymatoidal ideals generalizes ideals of Veronese type greatly. Two special types of PLP-polymatroidal ideals were studied in [11] and it was shown among others that $\operatorname{depth}\left(S / I^{k}\right)$ and $\operatorname{Ass}\left(S / I^{k}\right)$ become constant at the same $k$ for such ideals $I$.

We call the PLP-polymatroidal ideal of type $(\mathbf{a}, \mathbf{b} \mid \boldsymbol{\alpha}, \boldsymbol{\beta})$ to be basic if $\mathbf{a}=0$. Since every PLP-polymatroidal ideal is the product of a basic PLP-polymatroidal ideal and a monomial, we will only consider basic PLP-polymatroidal ideals hereafter.

Lemma 3.1. Assume that $\mathbf{b}, \boldsymbol{\alpha}, \boldsymbol{\beta}$ satisfy the conditions stated before and assume further that $\mathbf{a}=\mathbf{0}$. The system (1) of inequalities has an integral solution if and only if $\beta_{i}+b_{i+1}+\cdots+b_{j} \geq \alpha_{j}$ for any pair $i, j$ with $1 \leq i \leq j \leq n$.

Proof. Let $\mathbf{u}=\left(u_{1}, \ldots, u_{n}\right)$ be an integral solution of (1). Then for any $i, j$ with $1 \leq i \leq j \leq n$, we have $\alpha_{j} \leq\left(u_{1}+\cdots+u_{i}\right)+u_{i+1}+\cdots+u_{j} \leq \beta_{i}+b_{j+1}+\cdots+b_{n}$. This proves the necessity.

Conversely, assume that $\beta_{i}+b_{i+1}+\cdots+b_{j} \geq \alpha_{j}$ for any $i, j$ with $1 \leq i \leq j \leq n$. Then we set $u_{1}=\beta_{1}$, and

$$
u_{j+1}=\min \left\{\beta_{i}+b_{i+1}+\cdots+b_{j+1} \mid i \leq j+1\right\}-\left(u_{1}+u_{2}+\cdots+u_{j}\right)
$$

for $j=1, \ldots, n-1$. It is enough to show that $\mathbf{u}:=\left(u_{1}, \ldots, u_{n}\right)$ is an integral solution of (11).

Since $u_{1}+u_{2}+\cdots+u_{j}=\min \left\{\beta_{i}+b_{i+1}+\cdots+b_{j} \mid i \leq j\right\}$, it follows that $\alpha_{j} \leq$ $u_{1}+u_{2}+\cdots+u_{j} \leq \beta_{j}$ for $j=1, \ldots, n$. In addition, we have

$$
0 \leq u_{j+1}=\min \left\{u_{1}+u_{2}+\cdots+u_{j}+b_{j+1}, \beta_{j+1}\right\}-u_{1}+u_{2}+\cdots+u_{j} \leq b_{j+1}
$$

for $j=1, \ldots, n-1$. Here, to see $u_{j+1} \geq 0$, we need the assumption that $\beta_{j+1} \geq \beta_{j}$. Hence, the vector $\mathbf{u}$ is indeed an integral solution of (1).

Remark 3.2. In view of the proof of Lemma 3.1, we see that it still holds if the condition that $\alpha_{1} \leq \alpha_{2} \leq \cdots \leq \alpha_{n}$ is dropped.

In what follows, we keep the assumptions on $\mathbf{b}=\left(b_{1}, \ldots, b_{n}\right), \boldsymbol{\alpha}=\left(\alpha_{1}, \ldots, \alpha_{n}\right), \boldsymbol{\beta}=$ $\left(\beta_{1}, \ldots, \beta_{n}\right)$ stated before. In particular, $\alpha_{n}=\beta_{n}=d$.

Proposition 3.3. Let I be a basic PLP-polymatroidal ideal of type

$$
\left(\mathbf{0},\left(b_{1}, \ldots, b_{n}\right) \mid\left(\alpha_{1}, \ldots, \alpha_{n}\right),\left(\beta_{1}, \ldots, \beta_{n}\right)\right) .
$$


Then $\operatorname{soc}(I)$ is a basic PLP-polymatroidal ideal of type

$$
\left(\mathbf{0},\left(b_{1}-1, \ldots, b_{n}-1\right) \mid\left(\alpha_{1}, \ldots, \alpha_{n-1}, \alpha_{n}-1\right),\left(\beta_{1}-1, \ldots, \beta_{n}-1\right)\right) .
$$

Proof. We use $\mathbf{u} \in(1)$ to denote the condition that $\mathbf{u}$ is an integral solution of the system (11) for any $\mathbf{u} \in \mathbb{Z}_{+}^{n}$. Note that $a_{1}=\cdots=a_{n}=0$ in (11) by our assumption.

Denote by $J$ the PLP-polymatroidal ideal of type (*). We need to show $\operatorname{soc}(I)=$ $J$. Let $\mathbf{x}^{\mathbf{v}}$ be a minimal generator of $\operatorname{soc}(I)$. Then $v_{1}+\cdots+v_{n}=d-1$ and $\mathbf{v}+\mathbf{e}_{\mathbf{i}} \in(1)$ for any $i \in[n]$, where $\mathbf{e}_{\mathbf{1}}, \ldots, \mathbf{e}_{\mathbf{n}}$ is the canonical basis of $\mathbb{Z}_{+}^{n}$. Fix $2 \leq i \leq n-1$. Since $\mathbf{v}+\mathbf{e}_{\mathbf{n}} \in(\mathbb{1})$, we have $v_{1}+\cdots+v_{i}=\left(\mathbf{v}+\mathbf{e}_{\mathbf{n}}\right)([i]) \geq \alpha_{i}$. Here, for a vector $\mathbf{u}=\left(u_{1}, \ldots, u_{n}\right) \in \mathbb{Z}_{+}^{n}, \mathbf{u}([i])$ means the number $u_{1}+\cdots+u_{i}$. On the other hand, we have $v_{1}+\cdots+v_{i}=\left(\mathbf{v}+\mathbf{e}_{\mathbf{i}}\right)([i])-1 \leq \beta_{i}-1$, since $\mathbf{v}+\mathbf{e}_{\mathbf{i}} \in$ (1). Thus, $\alpha_{i} \leq v_{1}+\cdots+v_{i} \leq \beta_{i}-1$ for $i=2, \ldots, n-1$. It is also clear that $0 \leq v_{i} \leq b_{i}-1$ for $i=1, \ldots, n$. This implies $\operatorname{soc}(I) \subseteq J$.

Conversely, if $\mathbf{x}^{\mathbf{v}}$ is a minimal generator of $J$, then it is routine to check that $\mathbf{v}+\mathbf{e}_{\mathbf{i}} \in$ (1) for all $i=1, \ldots, n$. Hence $J \subseteq \operatorname{soc}(I)$, as desired.

Corollary 3.4. Let I be a basic PLP-polymatroidal ideal of type

$$
\left(\mathbf{0},\left(b_{1}, \ldots, b_{n}\right) \mid\left(\alpha_{1}, \ldots, \alpha_{n}\right),\left(\beta_{1}, \ldots, \beta_{n}\right)\right) .
$$

Then $\operatorname{depth}(S / I)=0$ if and only if the following inequalities hold:

$$
\begin{aligned}
& b_{i} \geq 1 \quad \text { for all } i=1, \ldots, n ; \\
& \alpha_{i} \leq \beta_{i}-1 \quad \text { for } \quad i=2, \ldots, n-1 ; \\
& \beta_{i}+b_{i+1}+\cdots+b_{j} \geq \alpha_{j}+j-i+1 \quad \text { for all } 1 \leq i \leq j \leq n-1 ; \\
& \beta_{i}+b_{i+1}+\cdots+b_{n} \geq d+n-i \quad \text { for all } 1 \leq i \leq n .
\end{aligned}
$$

Proof. Since $\operatorname{depth}(S / I)=0$ if and only if $\operatorname{soc}(I) \neq 0$, this result follows immediately by Proposition 3.3 together with Lemma 3.1 .

We now clarify when $\operatorname{Soc}(I) \neq 0$ in the case that $I$ is a basic PLP-polymatroidal ideal.

Corollary 3.5. Let I be a basic PLP-polymatroidal ideal of type

$$
\left(\mathbf{0},\left(b_{1}, \ldots, b_{n}\right) \mid\left(\alpha_{1}, \ldots, \alpha_{n}\right),\left(\beta_{1}, \ldots, \beta_{n}\right)\right) .
$$

Then the following statements are equivalent:

(a) $\operatorname{Soc}^{*}(I) \neq 0$;

(b) $S / I^{m}$ has limit depth zero, namely, $\lim _{m \rightarrow \infty}\left(S / I^{m}\right)=0$;

(c) $\ell(I)=n$;

(d) the following inequalities hold at the same time:

$$
\begin{aligned}
& b_{i} \geq 1 \quad \text { for } \quad i=1, \ldots, n \\
& \alpha_{i} \leq \beta_{i}-1 \quad \text { for } \quad i=2, \ldots, n-1 ; \\
& \beta_{i}+b_{i+1}+\cdots+b_{j}>\alpha_{j} \text { for } \quad 1 \leq i<j \leq n .
\end{aligned}
$$

Proof. $(a) \Leftrightarrow(b)$ is obvious and $(b) \Leftrightarrow(c)$ follows from [9, Corollary 2.5].

$(b) \Leftrightarrow(d)$ According to [11, Proposition 2.10], $I^{m}$ is a basic PLP-polymatroidal ideal of type $(\mathbf{0}, m \mathbf{b} \mid m \boldsymbol{\alpha}, m \boldsymbol{\beta})$. From this as well as Corollary 3.4, the result follows. 
Example 3.6. Let $t \geq 2$ and let $I=\left(x^{t}, x y^{t-2} z, y^{t-1} z\right) \subseteq S=K[x, y, z]$. Then $\operatorname{depth}\left(S / I^{n}\right)$ is 1 if $n \leq t-1$ and is 0 if $n \geq t$, according to [13, Proposition 2.5]. This implies $\operatorname{Soc}^{*}(I)$ is generated in degrees $\geq t-1$.

Example 3.6 demonstrates that $\operatorname{Soc}^{*}(I)$ may be generated in degrees far larger than $n$. But in the case that $I$ is a PLP-polymatroidal ideal, the situation is different.

Theorem 3.7. Let I be a PLP-polymatroidal ideal of type

$$
\left(\mathbf{0},\left(b_{1}, \ldots, b_{n}\right) \mid\left(\alpha_{1}, \ldots, \alpha_{n}\right),\left(\beta_{1}, \ldots, \beta_{n}\right)\right) .
$$

Then $\operatorname{Soc}^{*}(I)$ and $\operatorname{Soc}(I)$ are generated in degrees $<n$.

Proof. Since $\operatorname{Soc}(I) \cong \operatorname{Soc}^{*}(I) / \mathfrak{m} \operatorname{Soc}^{*}(I)$, we only need to prove $\operatorname{Soc}^{*}(I)$ are generated in degrees $<n$. By [11, Proposition 2.10] and Proposition 3.3, we see that $\operatorname{soc}\left(I^{\ell}\right)$ is of type

$$
\left(\mathbf{0},\left(\ell b_{1}-1, \ldots, \ell b_{n}-1\right) \mid\left(\ell \alpha_{1}, \ldots, \ell \alpha_{n-1}, \ell \alpha_{n}-1\right),\left(\ell \beta_{1}-1, \ldots, \ell \beta_{n}-1\right)\right) .
$$

It is enough to show that $\operatorname{soc}\left(I^{k+1}\right) \subseteq I \operatorname{soc}\left(I^{k}\right)$ for all $k \geq n-1$. Let $\mathbf{u}=\left(u_{1}, \ldots, u_{n}\right)$ be a vector in $\mathbb{Z}_{+}^{n}$ such that $\mathbf{x}^{\mathbf{u}}$ is a minimal generator of $\operatorname{soc}\left(I^{k+1}\right)$. Then we write $u_{i}=(k+1) s_{i}+t_{i}$ with $0 \leq t_{i} \leq k$ for every $i \in[n]$. We want to find a suitable vector $\left(\lambda_{1}, \ldots, \lambda_{n}\right) \in \mathbb{Z}_{+}^{n}$ such that $\mathbf{x}^{\mathbf{w}}$ and $\mathbf{x}^{\mathbf{v}}$ belong to $G(I)$ and $G\left(\operatorname{soc}\left(I^{k}\right)\right)$ respectively, where $\mathbf{w}:=\left(s_{1}+\lambda_{1}, \ldots, s_{n}+\lambda_{n}\right)$ and $\mathbf{v}:=\left(k s_{1}+t_{1}-\lambda_{1}, \ldots, k s_{n}+t_{n}-\lambda_{n}\right)$. To this end, we may write $\mathbf{w}:=\left(s_{1}+\lambda_{1}, \ldots, s_{n}+\lambda_{n}\right)$ and $\mathbf{v}:=\left(k s_{1}+t_{1}-\lambda_{1}, \ldots, k s_{n}+t_{n}-\lambda_{n}\right)$ with $\left(\lambda_{1}, \ldots, \lambda_{n}\right) \in \mathbb{Z}_{+}^{n}$ undetermined.

Since $(k+1) s_{i}+t_{i} \leq(k+1) b_{i}-1$, we have $s_{i} \leq b_{i}-\frac{1+t_{i}}{k+1}$ and so

$$
\begin{array}{r}
w_{i} \leq b_{i}-\frac{1+t_{i}}{k+1}+\lambda_{i} \quad \text { for } \quad i=1, \cdots, n \\
v_{i} \leq k b_{i}-\frac{k\left(1+t_{i}\right)}{k+1}+t_{i}-\lambda_{i} \text { for } \quad i=1, \cdots, n
\end{array}
$$

Set $\Lambda_{i}=\lambda_{1}+\cdots+\lambda_{i}$ for $i=1, \ldots, n$. For $i=2, \ldots, n-1$, since

$$
(k+1) \alpha_{i} \leq(k+1)\left(s_{1}+\cdots+s_{i}\right)+t_{1}+\cdots+t_{i} \leq(k+1) \beta_{i}-1,
$$

it follows that

$$
\alpha_{i}-\frac{t_{1}+\cdots+t_{i}}{k+1} \leq s_{1}+\cdots+s_{i} \leq \beta_{i}-\frac{1+t_{1}+\cdots+t_{i}}{k+1} .
$$

Hence, for $i=2, \ldots, n-1$, we have

$$
\begin{gathered}
\alpha_{i}-\frac{t_{1}+\cdots+t_{i}}{k+1}+\Lambda_{i} \leq w_{1}+\cdots+w_{i} \leq \beta_{i}-\frac{1+t_{1}+\cdots+t_{i}}{k+1}+\Lambda_{i}, \\
k \alpha_{i}+\frac{t_{1}+\cdots+t_{i}}{k+1}-\Lambda_{i} \leq v_{1}+\cdots+v_{i} \leq k \beta_{i}+\frac{t_{1}+\cdots+t_{i}-k}{k+1}-\Lambda_{i} .
\end{gathered}
$$


From the inequalities (2) and (3), it is not difficult to see that if

$$
\begin{aligned}
& \Lambda_{n}=\frac{t_{1}+\cdots+t_{n}+1}{k+1}=\beta_{n}-s_{1}-\cdots-s_{n} \\
& \frac{t_{1}+\cdots+t_{i}}{k+1}-\frac{k}{k+1}<\Lambda_{i}<1+\frac{t_{1}+\cdots+t_{i}}{k+1}, \quad i=2, \ldots, n-1 ; \\
& \frac{t_{i}-k}{k+1}<\lambda_{i}<1+\frac{1+t_{i}}{k+1}, \quad i=1, \ldots, n .
\end{aligned}
$$

then $\mathbf{x}^{\mathbf{w}} \in G(I)$ and $\mathbf{x}^{\mathbf{v}} \in G\left(\operatorname{soc}\left(I^{k}\right)\right)$. In what follows, we show that such $\lambda_{i}$ 's exist.

Note that the inequalities in (6) are equivalent to

$$
\lambda_{i}=1 \quad \text { if } \quad t_{i}=k \quad \text { and } \quad 0 \leq \lambda_{i} \leq 1 \quad \text { if } \quad 0 \leq t_{i} \leq k-1 .
$$

Let $[a]$ denote the largest integer $<a$ and $\lfloor a\rfloor$ the largest integer $\leq a$. Under these notations, the above system of inequalities (44), (5), (6) is equivalent to the following system:

$$
\left\{\begin{array}{l}
\lambda_{i}=1 \quad \text { if } \quad t_{i}=k \quad \text { and } \quad 0 \leq \lambda_{i} \leq 1 \quad \text { if } \quad 0 \leq t_{i} \leq k-1 \\
\left\lfloor\frac{t_{1}+\cdots+t_{i}}{k+1}-\frac{k}{k+1}\right\rfloor \leq \lambda_{1}+\cdots+\lambda_{i} \leq 1+\left[\frac{t_{1}+\cdots+t_{i}}{k+1}\right] \quad i=1,2, \ldots, n-1 \\
\lambda_{1}+\cdots+\lambda_{n}=\frac{t_{1}+\cdots+t_{n}+1}{k+1} .
\end{array}\right.
$$

Set $\varepsilon_{i}=\lambda_{i}-1$ if $t_{i}=k$ and $\varepsilon_{i}=\lambda_{i}$ otherwise. Let $c_{i}$ denote the number $\left|\left\{1 \leq j \leq i \mid t_{j}=k\right\}\right|$. Then the system (17) can be rewritten as:

$$
\left\{\begin{array}{l}
\varepsilon_{i}=0 \quad \text { if } \quad t_{i}=k \quad \text { and } \quad 0 \leq \varepsilon_{i} \leq 1 \quad \text { if } \quad 0 \leq t_{i} \leq k-1 \\
\max \left\{\left\lfloor\frac{t_{1}+\cdots+t_{i}}{k+1}-\frac{k}{k+1}\right\rfloor-c_{i}, 0\right\} \leq \varepsilon_{1}+\cdots+\varepsilon_{i} \leq 1+\left[\frac{t_{1}+\cdots+t_{i}}{k+1}\right]-c_{i} \\
\varepsilon_{1}+\cdots+\varepsilon_{n}=\frac{t_{1}+\cdots+t_{n}+1}{k+1}-c_{n}
\end{array}\right.
$$

Since $k \geq n-1 \geq c_{n}-1$, it follows that $\frac{t_{1}+\cdots+t_{n}+1}{k+1}-c_{n} \geq \frac{c_{n} k+1}{k+1}-c_{n}=\frac{1-c_{n}}{k+1}>-1$ and so

$$
\frac{t_{1}+\cdots+t_{n}+1}{k+1}-c_{n} \geq 0
$$

because $\frac{t_{1}+\cdots+t_{n}+1}{k+1}-c_{n}$ is an integer. Similarly, we have

$$
1+\left[\frac{t_{1}+\cdots+t_{i}}{k+1}\right]-c_{i} \geq 0 \quad \text { for } \quad i=1, \ldots, n-1
$$

For $i=1, \cdots, n$, we set

$$
h_{i}=\min \left\{\frac{t_{1}+\cdots+t_{n}+1}{k+1}-c_{n}, 1+\left[\frac{t_{1}+\cdots+t_{j}}{k+1}\right]-c_{j}, j=i, \ldots, n-1\right\} .
$$

Also we denote $g_{n}=\frac{t_{1}+\cdots+t_{n}+1}{k+1}-c_{n}$, and $g_{i}=\left\lfloor\frac{t_{1}+\cdots+t_{i}}{k+1}-\frac{k}{k+1}\right\rfloor-c_{i}$ for $i=1, \ldots, n-1$. Then $h_{n} \geq h_{n-1} \geq \cdots \geq h_{2} \geq h_{1} \geq 0$. We claim that $h_{i} \geq g_{i}$ for $i=1, \ldots, n$. For this, it is enough to prove that

$$
1+\left[\frac{t_{1}+\cdots+t_{j}}{k+1}\right]-c_{j} \geq\left\lfloor\frac{t_{1}+\cdots+t_{i}}{k+1}-\frac{k}{k+1}\right\rfloor-c_{i}
$$


for $1 \leq i \leq j \leq n$. Let $s=c_{j}-c_{i}$. Then, since $k \geq n-1 \geq s$, we have

$$
\begin{gathered}
1+\left[\frac{t_{1}+\cdots+t_{j}}{k+1}\right] \geq \frac{t_{1}+\cdots+t_{i}+s k}{k+1} \\
\geq \frac{t_{1}+\cdots+t_{i}+s k+s-k}{k+1} \geq \frac{t_{1}+\cdots+t_{i}-k}{k+1}+s .
\end{gathered}
$$

This proves the desired inequality (9) and so our claim follows.

Set $e_{i}=0$ if $t_{i}=k$ and $e_{i}=1$ otherwise. Note that $g_{1}=0$ and $e_{1}=h_{1}$. Hence, the system (8) can be rewritten as follows.

$$
\left\{\begin{array}{l}
0 \leq \varepsilon_{i} \leq e_{i}, \quad i=1, \ldots, n \\
\max \left\{g_{i}, 0\right\} \leq \varepsilon_{1}+\cdots+\varepsilon_{i} \leq h_{i}, \quad i=2, \ldots, n
\end{array}\right.
$$

In view of Lemma 3.1 and Remark 3.2, to prove that the system (10) has an integral solution, it is enough to show that $h_{i}+e_{i+1}+\cdots+e_{j} \geq g_{j}$ for all pairs $n \geq j \geq i \geq 1$. Fix $i, j$ with $n \geq j \geq i \geq 1$. If $i=n$, there is nothing to prove. If $i \leq n-1$ and

$$
h_{i}=\min \left\{\frac{t_{1}+\cdots+t_{n}+1}{k+1}-c_{n}, 1+\left[\frac{t_{1}+\cdots+t_{\ell}}{k+1}\right]-c_{\ell}, \ell=j, \ldots, n-1\right\},
$$

then $h_{i}=h_{j} \geq g_{j}$ and we are done. So it remains to consider the case when $1 \leq i<n$ and when $h_{i}=1+\left[\frac{t_{1}+\cdots+t_{\ell}}{k+1}\right]-c_{\ell}$ for some $\ell \in\{i, \ldots, j-1\}$. Note that $e_{i+1}+\cdots+e_{j}=j-i-c_{j}+c_{i}$. Hence it is enough to show that

$$
1+\left[\frac{t_{1}+\cdots+t_{\ell}}{k+1}\right]-c_{\ell}+j-i-\left(c_{j}-c_{i}\right) \geq\left\lfloor\frac{t_{1}+\cdots+t_{j}}{k+1}-\frac{k}{k+1}\right\rfloor-c_{j} .
$$

Since $c_{\ell}-c_{i} \leq \ell-i$, it is enough to prove

$$
1+\left[\frac{t_{1}+\cdots+t_{\ell}}{k+1}\right]+j-\ell \geq\left\lfloor\frac{t_{1}+\cdots+t_{j}}{k+1}-\frac{k}{k+1}\right\rfloor .
$$

But this is clear and so our proof is completed.

A finitely generated graded module $M$ is called equi-generated if it generated in a single degree, namely, $M$ is generated in degree $d$, where $d=\min \left\{i \in \mathbb{Z}: M_{i} \neq 0\right\}$. Finally, we discuss when $\operatorname{Soc}^{*}(I)$ is equi-generated when $I$ is an ideal of Veronese type.

Let $I=I_{\left(a_{1}, \ldots, a_{n}\right), d}$ be an ideal of Veronese type (cf. [9]) and let $\rho$ be the rank function of the polymatroid corresponding to $I$ (see [6] or [7] for the definition and basic properties of rank functions). Denote $\left(a_{1}, \ldots, a_{n}\right)$ by $\mathbf{a}$. Then we have the following observations.

(1) depth $S / I=0$ if and only if $a_{i} \geq 1$ for all $i \in[n]$ and $\sum_{i \in[n]} a_{i}-d \geq n-1$;

(2) $\operatorname{Soc}^{*}(I) \neq 0$ if and only if $a_{i} \geq 1$ for all $i \in[n]$ and $\sum_{i \in[n]} a_{i}>d$;

(3) $\rho(A)=\min \{\mathbf{a}(A), d\}$ for any $A \subseteq[n]$, here $\mathbf{a}(A)=\sum_{i \in A} a_{i}$;

(4) $I^{k}=I_{k \mathbf{a}, k d}$ for all $k \geq 1$;

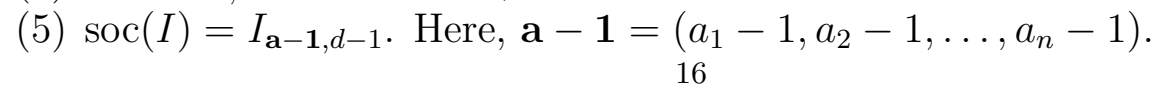


Proposition 3.8. Let $I=I_{\left(a_{1}, \ldots, a_{n}\right), d}=I_{\mathbf{a}, d}$. Suppose that $a_{i} \geq 1$ for all $i \in[n]$ and $\mathbf{a}([n])>d$. Then the following statements are equivalent:

(1) $\operatorname{Soc}(I)$ is equi-generated;

(2) $\operatorname{Soc}^{*}(I)$ is equi-generated;

(3) For any $A \subseteq[n]$ with $\mathbf{a}(A)>d$, one has $k_{0}(\mathbf{a}(A)-d) \geq|A|-1$. Here $k_{0}$ is the least number $k$ such that $k(\mathbf{a}([n])-d) \geq n-1$ and $|A|$ denotes the number of elements of $A$.

Proof. (1) $\Longleftrightarrow(2)$ This follows from Nakayama Lemma as well as the isomorphism $\operatorname{Soc}(I) \cong \operatorname{Soc}^{*}(I) / \mathfrak{m} \operatorname{Soc}^{*}(I)$.

$(2) \Rightarrow(3)$ We only need to prove that if there exits $A \subseteq[n]$ such that $\mathbf{a}(A)>d$ and $k_{0}(\mathbf{a}(A)-d)<|A|-1$, then $I \operatorname{soc}\left(I^{k_{0}}\right) \neq \operatorname{soc}\left(I^{k_{0}+1}\right)$.

Let $\rho_{1}$ and $\rho_{2}$ be the rank functions of $I \operatorname{soc}\left(I^{k_{0}}\right)$ and $\operatorname{soc}\left(I^{k_{0}+1}\right)$ respectively. Then

$$
\rho_{1}(A)=\min \{\mathbf{a}(A), d\}+\min \left\{k_{0} \mathbf{a}(A)-|A|, k_{0} d-1\right\}=d+k_{0} \mathbf{a}(A)-|A| .
$$

Here, the second equality follows by the choice of the subset $A$. On the other hand, $\rho_{2}(A)=\min \left\{\left(k_{0}+1\right) \mathbf{a}(A)-|A|,\left(k_{0}+1\right) d-1\right\}$. We claim that

$$
d+k_{0} \mathbf{a}(A)-|A| \notin\left\{\left(k_{0}+1\right) \mathbf{a}(A)-|A|,\left(k_{0}+1\right) d-1\right\} .
$$

In fact, if $d+k_{0} \mathbf{a}(A)-|A|=\left(k_{0}+1\right) \mathbf{a}(A)-|A|$ then $\mathbf{a}(A)=d$. This is contradicted to our assumption on $A$; if $d+k_{0} \mathbf{a}(A)-|A|=\left(k_{0}+1\right) d-1$, then $k_{0}(\mathbf{a}(A)-d)=|A|-1$. This is also contradicted to our assumption on $A$. Thus, our claim has been proved. From this claim it follows that $\rho_{1}(A) \neq \rho_{2}(A)$ and $I \operatorname{soc}\left(I^{k_{0}}\right) \neq \operatorname{soc}\left(I^{k_{0}+1}\right)$. Thus $\operatorname{Soc}^{*}(I)$ is not equi-generated.

$(3) \Rightarrow(2)$ Assume (3) holds. We will prove $I \operatorname{soc}\left(I^{k_{0}}\right)=\operatorname{soc}\left(I^{k_{0}+1}\right)$ for all $k \geq k_{0}$. Fix $k \geq k_{0}$ and denote by $\rho_{1}$ and $\rho_{2}$ the rank functions of $I \operatorname{soc}\left(I^{k_{0}}\right)$ and $\operatorname{soc}\left(I^{k_{0}+1}\right)$ respectively. It is clear that

$$
\rho_{1}(A)=\min \{\mathbf{a}(A), d\}+\min \{k \mathbf{a}(A)-|A|, k d-1\}
$$

and

$$
\rho_{2}(A)=\min \{(k+1) \mathbf{a}(A)-|A|,(k+1) d-1\} .
$$

We now check that $\rho_{1}(A)=\rho_{2}(A)$ for all $\emptyset \neq A \subseteq[n]$.

Fix $\emptyset \neq A \subseteq[n]$. If $\mathbf{a}(A) \leq d$, then $\ell \mathbf{a}(A)-|A| \leq \ell d-1$ for $\ell=k, k+1$, and so $\rho_{1}(A)=(k+1) \mathbf{a}(A)-|A|=\rho_{2}(A)$.

If $\mathbf{a}(A)>d$, then $\ell \mathbf{a}(A)-|A| \geq \ell d-1$ for $\ell=k, k+1$ by (3). This implies $\rho_{1}(A)=(k+1) d-1=\rho_{2}(A)$. Therefore, $\rho_{1}=\rho_{2}$ and $I \operatorname{soc}\left(I^{k_{0}}\right)=\operatorname{soc}\left(I^{k_{0}+1}\right)$ for all $k \geq k_{0}$, as desired.

In general $\operatorname{Soc}^{*}(I)$ is not equi-generated even if $I$ is an ideal of Veronese type.

Example 3.9. Let $I=I_{(3,3,1,2), 6}$. Then $\operatorname{soc}(I) \neq 0$ and so $k_{0}=1$. Let $A=\{1,2,3\}$. Then $\mathbf{a}(A)=7>6=d$, but $k_{0}(\mathbf{a}(A)-6)<2=|A|-1$. Hence $\operatorname{Soc}^{*}(I)$ is not equi-generated by Proposition 3.8 .

acknowledgement: The paper was written while the first author and the third author were visiting the Department of Mathematics of University Duisburg-Essen Germany. They want to express their thanks for its hospitality. This research is supported by NSFC (No. 11971338). 


\section{REFERENCES}

[1] J. Abbott and A. M. Bigatti, a C++ library for doing Computations in Commutative Algebra, Available at http://cocoa.dima.unige.it/cocoalib.

[2] A. Banerjee The regularity of powers of edge ideals. J. Algebraic Combin., 41(2015):303-321

[3] J. Chen, S. Morey, A. Sung, The stable set of associated primes of the ideal of a graph, Rocky Mountain Journal of Mathematics, 32(2002):71-89

[4] H. T. Hà, N. V. Trung, T. N. Trung, Depth and regularity of powers of sums of ideals, Math. Z. (2016) 282:819-838

[5] J. Herzog, T. Hibi, Bounding the socles of powers of squarefree monomial ideals, In: Commutative algebra and noncommutative e algebraic geometry. Vol. II, 2015, 223-229.

[6] J. Herzog, T. Hibi, Discrete polymatroids, J. Algebraic Combin. 16 (2002) 239-268.

[7] J. Herzog, T. Hibi, Monomial Ideals, Grad. Texts in Math., vol. 260, Springer, New York, 2011.

[8] J. Herzog, A. Asloob Qureshi, Persistence and stability properties of powers of ideals, Journal of Pure and Applied Algebra 219 (2015) 530-542

[9] J. Hezog, A. Rauf, M. Vladoiu, The stable set of associated prime ideals of a polymatroidal ideal, J. Algebr. Comb (2013) 37:289-312

[10] H.M. Lam, N.V. Trung, Associated primes of powers of edge ideals and ear decompositions of graphs, Transactions of the American Mathematical Society, 372(5), 2019, 3211-3236.

[11] Dancheng Lu, Discrete polymatroids satisfying a stronger symmetric exchange property, Journal of Algebra, 490(2017), 21-54.

[12] J. Martínez-Bernal, S. Morey, R.H. Villarreal, Associated primes of powers of edge ideals, Collect. Math. 63 (2012) 361-374.

[13] K. Matsuda, T. Suzuki and A. Tsuchiya, Nonincreasing depth functions of monomial ideals, Glasg. Math. J. 60 (2018), no. 2, 505-511.

[14] L. J. Ratliff Jr. On prime divisors of $I^{n}$, $n$ large, Michigan Math. J., 23 (1976), 337-352.

[15] J. Schweig, Toric ideals of lattice path matroids and polymatroids, J. Pure Appl. Algebra 215 (2011) 2660-2665.

[16] T. N. Trung, Stability of depths of powers of edge ideals, Journal of Algebra 452(2016)157-187

Lizhong Chu, School of Mathematical Sciences, Soochow University, P.R.China E-mail address: chulizhong@suda.edu.cn

Jürgen Herzog, Fachbereich Mathematik, Universität Duisburg-Essen, Campus Essen, 45117 Essen, Germany

E-mail address: juergen.herzog@uni-essen.de

Dancheng Lu, School of Mathematical Sciences, Soochow University, P.R.China E-mail address: ludancheng@suda.edu.cn 